OPEN ACCESS

Edited by:

Xuyao Zhang,

Fudan University, China

Reviewed by:

Luciano Mutti,

Temple University, United States

*Correspondence:

Albert D. Donnenberg donnenbergad@upmc.edu

Vera S. Donnenberg

donnenbergvs@upmc.edu

Specialty section:

This article was submitted to

Cancer Immunity

and Immunotherapy,

a section of the journal

Frontiers in Immunology

Received: 30 December 2021 Accepted: 20 January 2022 Published: 11 February 2022

Citation:

Donnenberg VS, Wagner PL, Luketich JD, Bartlett $D L$ and

Donnenberg AD (2022) Localized Intra-Cavitary Therapy to Drive Systemic Anti-Tumor Immunity.

Front. Immunol. 13:846235. doi: 10.3389/fimmu.2022.846235

\section{Localized Intra-Cavitary Therapy to Drive Systemic Anti-Tumor Immunity}

\author{
Vera S. Donnenberg ${ }^{1,2,3^{*}}$, Patrick L. Wagner ${ }^{4,5}$, James D. Luketich ${ }^{1,3}$, David L. Bartlett ${ }^{4,5}$ \\ and Albert D. Donnenberg ${ }^{1,2,6 *}$ \\ ${ }^{1}$ University of Pittsburgh Medical Center (UPMC) Hillman Cancer Centers, Pittsburgh, PA, United States, ${ }^{2}$ McGowan \\ Institute for Regenerative Medicine, Pittsburgh, PA, United States, ${ }^{3}$ Department of Cardiothoracic Surgery, University of \\ Pittsburgh School of Medicine, Pittsburgh, PA, United States, ${ }^{4}$ Surgical Oncology, Allegheny Health Network Cancer \\ Institute, Pittsburgh, PA, United States, ${ }^{5}$ College of Medicine, Drexel University, Pittsburgh, PA, United States, ${ }^{6}$ Department \\ of Medicine, University of Pittsburgh School of Medicine, Pittsburgh, PA, United States
}

Metastasis to the pleural and peritoneal cavities is a common terminal pathway for a wide variety of cancers. This article explores how these unique environments both promote aggressive tumor behavior and suppresses anti-tumor immunity, and ways in which local delivery of protein therapeutics can leverage the contained nature of these spaces to a therapeutic advantage, achieving high intra-cavital concentrations while minimizing systemic toxicity.

Keywords: malignant pleural effusion, malignant ascites, local therapy, intratumoral, epithelial to mesenchymal transition (EMT), video assisted thoracoscopic surgery (VATS)

\section{INTRODUCTION}

Malignant pleural effusions (MPE) have a US incidence of more than 150,000 cases per year $(1,2)$ and a life expectancy measured in months (3). Likewise, patients with malignant peritoneal ascites (MPA) and peritoneal carcinomatosis also have abysmal survival (4) and poor quality of life (QOL (5),) with patients experiencing multiple hospitalizations for bowel obstruction and pain (6). Both MPE and MPA are common, painful, difficult to treat, and most importantly are uniformly fatal. Despite significant clinical progress in immuno-oncology, there has been almost no change in survival or quality of life for patients with MPE or MPA, which become the proximate cause of death in many cases of advanced cancer

\section{SUBSECTIONS}

\section{An Incomplete Understanding of the Pleural and Peritoneal Cavity Environments}

In states of normal health, the pleural and peritoneal cavities contain physiologic fluid with a dynamic array of immune cells and unique secretomes. In non-neoplastic pathologic states, fluid can accumulate in these cavities due either to transudative mechanisms (vascular pressure and decreased resorption), or exudative mechanisms (tissue inflammation and immune cell infiltration). These processes result in dramatic changes to the cavitary secretome and immune environment. Malignant effusions and ascites also exhibit similar shifts in the secretome and cellular infiltrate, 
but several additional cytokines and chemokines distinguish them from benign effusions (Figure 1A). The presence of additional malignancy-specific components notwithstanding, the combination of cytokines seen across the spectrum of benign and malignant conditions is predicted to drive both aggressive tumor behavior and polarize pleural immune cells away from tumor-specific immunity and instead, toward a maladaptive repair-and-regenerate mode. Understanding the interplay between the secretome and the resident cell populations will provide much needed foundation for the development of innovative immunotherapeutics targetable to the cavitary spaces and specific to the mechanisms relevant to malignant effusions and ascites.

\section{The Secretomic Signatures of Malignant Peritoneal Ascites and Pleural Effusions Are Indistinguishable}

One of the most striking features of Figure 1A, which contrasts the secretomes of ovarian cancer ascites and pleural effusions with that of benign pleural effusions, is the similarity between the cytokine and chemokine profiles in pleural and peritoneal malignancies. In the healthy cavitary spaces, a physiologic secretome and a complement of immune cells are dedicated to maintenance and repair of the mesothelial lining through a process involving the epithelial to mesenchymal transition (EMT, detachment, migration) and the mesenchymal to epithelial transition (MET, reattachment, re-epithelialization) (9). In malignant fluid, the environment is transformed into an inflammatory milieu that promotes wound healing and suppresses adaptive immune effector responses. As mentioned above, this tumor-related cavitary fluid environment has all the inflammatory components of benign effusions (IL-6, sIL-6R $\alpha$, CCL2, CXCL10, TGF $\beta$, CCL22, IL-8, IL-5 Figure 1), but includes additional tumor and endothelial growth factors, as well as a blunted effector cytokine response (IFN $\alpha 2)$ that is not seen in physiologic cavitary fluid or benign effusions.

\section{Effects of the Malignant Secretome on Mesothelial Maintenance, Intra-Cavitary Tumor and Anti-Tumor Responses}

Because the pleural and peritoneal spaces are lined by mesothelial cells, and because effusions and ascites result from a net inflow from the peripheral circulation, these cavities develop a unique cytokine environment. In the context of malignancy, the milieu of cytokines is predicted to play a maladaptive, tumor-supporting role, with negative effects not only in the cavitary environment, but systemically as well, promoting motility and solid organ metastasis. Figure 1B illustrates mechanisms by which the inflammatory cavitary secretome, initiated by tumor metastasis and supplemented by tumor-secreted cytokines, is predicted to establish an environment that promotes aggressive tumor behavior. These mechanisms include EMT, suppression of adaptive T-cell effector responses, and polarization of macrophages to support rather than oppose tumor growth. Although ovarian cancer is presented here as an example, the malignant cavitary secretome, and the resulting tumor pathobiology is common to a wide range of cancers $(7,8)$.

\section{A Rationale for Intra-Cavitary Therapy Using Immune-Oncology Drugs}

The physiologically isolated pleural and peritoneal environments provide ideal anatomical spaces for the localized administration of large protein drugs. The pleura as well as the peritoneum are lined with mesothelial cells joined by tight junctions (10) creating a unique and isolated cavitary environment. Unlike chemotherapeutics, high molecular weight immuno-oncology drugs remain concentrated when administered directly to these cavities, reaching a high target occupancy even with protein drugs with a narrow therapeutic index when administered systemically (11-14).

Local administration of therapeutics may be used to directly target the tumor, support local immune cells, and condition cancer associated stromal cells (15). Despite the net inflow of serous fluid, IgG levels are lower in pleural effusions (16) and peritoneal ascites than in the peripheral circulation. Thus, intravenous administration of protein therapeutics may not be the most effective way to achieve the necessary therapeutic levels within the cavitary spaces. Conversely, localized intra-cavitary administration of these therapeutics has been shown to result in low systemic exposure, and negligible on-target off-tumor effects, while reducing adverse events associated with systemic toxicity $(13,14)$. The same principle applies to intra-cavitary injection of RNA-based therapeutics (17, 18). This mode of administration is greatly facilitated by the use of minimally invasive surgical techniques and placement of indwelling catheters. While these catheters are traditionally used for palliative decompression of the cavitary spaces, they have been repurposed as drug delivery devices, for example the instillation of pleurodesis agents or cytotoxic chemotherapy. Video assisted thoracoscopic surgery [VATS (19)] or laparoscopic peritoneal catheter placement (20) can likewise be used to guide intracavital-intratumoral drug delivery for agents such as oncovaccines and mRNA therapeutics, which are injected directly into tumor foci. Further, indwelling catheters allow for iterative sampling of cavitary fluid to monitor the response to therapy, the impact on the cavitary immune environment, the pharmacokinetics and pharmacodynamics (PK/ $\mathrm{PD}$ ) of personalized drug dosing, and the real time determination of minimal anticipated biological effect levels [MABEL (21)]. Finally, anti-tumor effector responses initiated in the confines of the cavitary spaces would be expected to propagate systemically through the draining lymphatics, where they could combat solid organ metastases. Although most studies of single-agent intracavital immunotherapies have not measured effects on systemic immunity directly, the intrapleural (14) and intraperitoneal (13) experience with the bispecific anti-CD3/anti-EpCAM antibody catumaxomab is informative. Even though the systemic catumaxomab concentration was $<1 \%$ of the intracavitary concentration, both studies observed transient increases in serum transaminases, which were attributed to systemic cytokine release. Similarly, intracavitary IL-2 administration resulted in an increase in peripheral CD8 T cells expressing granzyme B (22) and in peripheral NK cell activity (12), and intracavitary administration of 
an adenovirus/interferon $\beta$ construct resulted in increased peripheral NK activity and anti-tumor antibodies (23).

In this manner, the cavitary spaces can be conceived of as 'bioreactors' into which novel immunotherapeutic agents could be instilled or injected intratumorally with the aim of selecting and stimulating effector cytotoxic $\mathrm{T}$ cells for cavitary and abscopal activity. We argue for local rather than systemic administration of protein-based therapies, since systemic administration may not achieve the necessary intracavitary therapeutic levels (11).

Intra-cavitary therapy can be considered as a special case of intra-tumoral therapy (reviewed in (24). Intra-tumoral immunotherapies have been explored extensively with the intention of altering the immune microenvironment of solid tumors to promote adaptive immunity. The peritoneal and pleural cavities are fluid-phase environments that may be more amenable to targeted manipulation. Although clusters of tumor cells are usually demonstrable in the fluid phase, the bulk of the lesions cake the mesothelium and adhere to internal organs, where they are bathed in the fluid phase.

\section{Effusions and Ascites as a Source for Adoptive Cellular Therapy}

Adoptive T-cell therapy using autologous tumor infiltrating lymphocytes (TIL) has been reported to induce salvage responses in a variety of refractory solid tumors (25). Conventionally, TIL therapy requires large-scale expansion of a small number of T-cells grown out from tumor tissue fragments stimulated with high dose IL-2 and anti-CD3 antibody. Since the expanded TIL depend on the continued presence of IL-2 for their survival, TIL infusion must be accompanied by repeated systemic administration of high dose IL-2, stopping only when doselimiting toxicity is reached. TIL infusion is often preceded by treatment with immunosuppressive chemotherapeutic agents such as cyclophosphamide and fludarabine to make space for the therapeutic cells. Therapeutic drainage of MPE frequently yields on the order of 0.25 to $0.5 \times 10^{6}$ pleural T cells $/ \mathrm{mL}$. In our experience, it is not unusual to drain a liter of fluid, yielding up to $5 \times 10^{8} \mathrm{~T}$ cells. Macrophages are also prevalent, constituting up to $50 \%$ of total nucleated MPE cells. Thus, in a single drainage it is often possible to obtain potentially therapeutic doses of pleural $\mathrm{T}$ cells following short-term activation or expansion. The ex vivo activated cells can be positively selected for CD45+ T cells and macrophages and then re-instilled into the pleural or peritoneal cavity. Potential advantages over conventionally expanded TIL include greatly simplified and rapid manufacture, potentially eliminating the requirement for systemic administration of toxic high dose IL-2 to ensure cell survival after reintroduction.

\section{Potential Drawbacks to Intra-Cavitary Therapeutics}

The principal problems facing intra-cavitary therapies are determining the most efficacious combination of therapeutics and the logistics associated with intra-cavitary and intratumoral administration. Additionally, the high cost of biologics, and particularly patient-specific cellular therapeutics must also be considered. There are a broad range of potential modalities that can be locally delivered, and within these modalities a variety of agents with similar targets or mechanisms of action, with many more in pre-clinical or early phase clinical trials $(24,26,27)$. The varied formulations and compatibilities of potentially useful therapeutics must be considered if they are to be coadministered through indwelling catheters. Some therapeutics will be administered into the cavities, but others will require precise intratumoral delivery, which is limited to accessible tumor. Drug retention at intratumoral injection sites poses an additional potential difficulty and must be addressed by choosing appropriate agents and vehicles. For example, intratumoral injection of liposomal IL-12 mRNA (28) is more likely to remain localized than injection of the cytokine itself. Unexpected toxicities resulting from localized immune hyperresponsiveness, or interference with normal tissue maintenance may also pose problems, especially if they are delayed. Quantification of responses will likely require objective criteria similar to the RECIST score for solid tumors (29).

Technical challenges to implementation of intra-cavitary therapy include the need for dedicated personnel and facilities, including those required for image guided drug delivery. For drug delivery protocols requiring general anesthesia, the ability to administer repeated doses will be limited. Toxicities specific to intraperitoneal immunotherapy may be anticipated based on the experience with intra-peritoneal IL-2 (30) and monoclonal antibody (31) therapy (pyrexia, abdominal pain, nausea/ vomiting). These toxicities may be cavity specific as they were far milder with intrapleural administration of the same cytokine $(12,32)$ or antibody (14).

Finally, maximizing benefit with respect to cost is a challenge that must be met if intracavitary therapy is to gain acceptance. Given the dire prognosis and current palliative approaches to cavitary malignancies, any therapeutic combination that can provide an objective increase in response rates and survival with improved quality of life may justify the current high cost of immunotherapeutics. However, once Phase I/II trials have been completed, it will be important to initiate therapy while patients still have acceptable performance status and limited disease burden (33).

\section{DISCUSSION}

Since tumors that metastasize to the pleura and peritoneum exist in an environment tailored to EMT and immune suppression, combination therapy directed toward conditioning the local environment as well as activating anti-tumor immunity is warranted. Figure 2A divides these goals into four categories that can be addressed with intra-cavitary and intratumoral therapies: 1) Turning cold tumors hot; 2) Increasing tumorassociated antigen presentation; 3) Supporting effector $\mathrm{T}$ cell responses; and 4) Conditioning the local environment to block EMT. 
Tumors can be made more immunogenic by inducing damage associated molecular patterns (DAMPS) with therapies such as radiation, or pathogen associated molecular patterns (PAMPS) via oncolytic virus therapy (35). Tumors may also constitutively express receptors for PAMPS and DAMPs (toll-like receptors, TLR), but their prognostic significance varies with disease and receptor type (36). Introduction of TLR ligands through natural infection of the pleura (empyema) has been associated with prolonged survival in patients with cancer metastatic to the pleura or lung cancer $(37,38)$. This response may be due to PAMP-associated repolarization of the local immune environment, with concomitant alternation of the cytokine profile and augmentation of tumor antigen presentation by resident macrophages and dendritic cells. Numerous attempts have been made to exploit TLR receptor agonists as single agent therapeutics with limited success. This does not rule out the possibility that they will be a highly effective adjuvant to other immune oncology interventions.

IL-12 plays a central role in inducing and maintaining Th1 Tcell polarization (39). The low levels of IL-12p70 in pleural and ascites fluid (Figure 1A) suggest that IL-12 can be a key therapeutic for conditioning the intra-cavital environment. Multiple clinical trials of IL-12 protein and IL-12 mRNA support its potential use in intra-cavitary therapy, where systemic adverse effects can be limited. IFN $\gamma$ promotes T-cell and NK effector cells both directly and indirectly. Like IL-12, local administration may be advantageous to achieve functional concentrations while limiting systemic toxicity. Finally, therapeutic $\mathrm{T}$ cells may be activated or expanded ex vivo for intra-cavitary administration, provided that they are not reintroduced into an immunosuppressive environment.
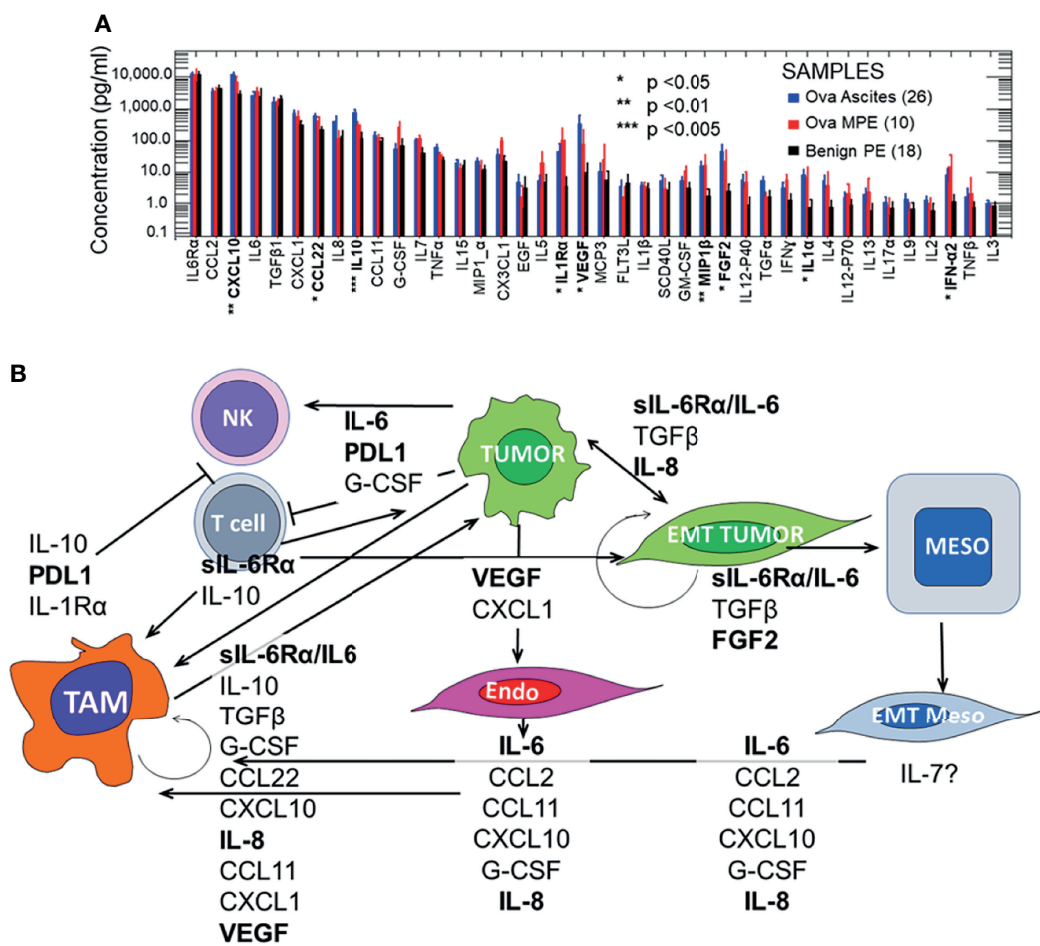

FIGURE 1 | Pleural and peritoneal secretomes in ovarian cancer and benign pleural effusions. Panel (A) Pleural and peritoneal secretomes are dominated by immunosuppressive and EMT tumor promoting cytokines and chemokines (IL-6/IL-6R $\alpha$, VEGF, IL-8, CXCL10, and IL-10). Data were log-transformed for analysis. There were no significant differences between analytes in ovarian ascites and malignant pleural effusions (MPE). Data for ovarian ascites and MPE were pooled and compared to Benign PE (Student's 2-tailed t test). Results were Bonferroni corrected for 40 comparisons. Statistically significant comparisons are indicated in bold typeface. Effusions and ascites were collected as anonymized medical waste under a University of Pittsburgh IRB exemptions (Nos. 0503126 and 0403111 ). Thirteen pleural effusions were collected from patients without pleural malignancy (11 with heart failure, 2 with asbestosis). The benign effusion data have been published previously $(7,8)$. Cytokines were quantified on the Luminex platform, using the Curiox LT-MX plate washer, Curiox DA-96 plates, the Luminex 200 System analyzer and XPonent data acquisition and analysis software. Cytokines were measured in $5 \mu \mathrm{L}$ of neat, clarified effusion using the MILLIPLEX MAP Human Cytokine/ Chemokine Magnetic Bead Panel - Premixed 38 Plex (Cat. No. HCYTMAG-60K-PX38), MILLIPLEX MAP Human TGF $\beta$ (Cat. No. TGFBMAG-64K-01) and IL-6R $\alpha$ from the Human Angiogenesis/Growth Factor Panel 2 (Cat. No. HANG2MAG-12K-01) kits, as previously published (Donnenberg et al., https://doi.org/10.18632/ oncotarget.27290). Panel (B) Effects of the malignant secretome on cavitary cell types and mesothelial maintenance. The 10 most prominent cytokines and chemokines (geometric mean $\geq 100 \mathrm{pg} / \mathrm{mL}$ ) in intracavitary ovarian cancer are shown, plus PDL1 which is expressed on intracavitary macrophages and must be addressed for successful intra-cavitary therapy. The variety of cell types, cytokines and chemokines involved, and the potential to amplify effects through autocrine and juxtacrine feedback loops justifies the need for multimodal intra-cavitary therapy. Potential therapeutic targets for which on- or off-label FDA approved agents are currently available are shown in bold typeface. 
A

Turn tumors hot by increasing TAA presentation

Intra-Tumoral TLR immune conditioning using PAMPs and DAMPs

- TLR agonists have been effectively used as "adjuvant" in anti-tumor immunity.

- Works across tumor types.

- Drives co-stimulation (increases GM-CSF), DC maturation, macrophage polarization.

- Stimulates IFNY production by T cells.

- Oncolytic viral therapy drives anti-tumor immunity by TLR-activation and viral-

tumor antigen expression.

- Localized radiation triggers profound DAMP release.

Maintain a TH1 environment

Localized sustained intra-/peri-tumoral IL-12 as the central mediator of TH1

environment

- Decreases T reg activity.

- Increases DC/Ag presentation.

- Supports DC trafficking to draining $L N$ and tertiary lymphoid structures.

- Increases cytotoxic CD4.

- Drives tumor-specific antibody production: Discovery of antibody-bound tumor neoantigens.

Support effector T cells

Localized intra-cavitary IFN $\gamma$ to promote sustained anti-tumor effector responses and TH1 cytokine cascade

- Support effectorT cells and NK cells.

- Recruit and support M1 macrophages.

- Repolarize T-regs to produce more IFN $Y$ and DC to produce IL-12.

- May increase PD1-PDL1 expression necessitating localized/systemic anti-PD therapy.

Condition intra-cavitary environment

High levels of secreted IL-6/IL-6R $\alpha$ in pleural and peritoneal fluids drive tumor EMT, invasion and polarize local immune responses to a "wound healing" state

- Block tumor EMT with anti-IL-6R $\alpha$ or anti-IL-6.

- Conditioning local environment away from "wound-healing" state.

- Prevent IL-6 induced cachexia.

Onco-immune targeting

To support the nascent anti-tumor response and PD-protect

- Anti-PD/PD-L: pembrolizumab, nivolumab, atezolizumab

- IFNp

- IL-2

- IL-12: MEDI1191/lipid nanoparticleformulated hIL-12 mRNA, hrlL-12 protein

- Anti-CTLA4: ipilimumab

- CD137/4-1BB: urelumab

- Patient specific TAA targeting: cellular and molecular/mRNA-based vaccines*, cavitaryderived TIL, and CAR-T,

- Anti-LAG3: relatlimab

- Anti-IL-10: LLL-3348, CRx-139
EMT and cavitary environment targeting

\section{To reverse the maladaptive milieu and EMT}

- LPS (TLR4) and other TLR agonists:

imiquimod (TLR7), resiquimod (TLR7/8)

- Anti-IL-6: siltuximab

- Anti-IL-6R $\alpha$ : tocilizumab, satralizumab, sarilumab

- Anti-VEGF: bevacizumab

- Anti-IL-8: HuMax-IL8 (BMS-986253), rivanicline

- Anti-TGF $\beta$ and integrin inhibitors: anti-TGFb antibodies (NIS793, SAR439459, LY3022859, AVID200) and TGF $\beta$ sequester GC1008/Fresolimumab, and anti-integrins: $\alpha v \beta 3, \alpha v \beta 5, \alpha v \beta 1$

- Oncolytic viral therapy*

- Anti-histamines, anti-IL-5: benralizumab, anti-IgE: omalizumab and anti-IL-4: dupilumab**

FIGURE 2 | Intra-cavitary therapeutics to drive systemic immunity and reverse EMT. Panel (A) Since tumors that metastasize to the pleural and peritoneum exist in an environment tailored to EMT and immune suppression, combination therapy directed toward conditioning the local environment as well as activating anti-tumor immunity is required. Panel (B) A list of potential therapeutics directed toward reversing tumor EMT, repolarizing the cavitary maladaptive milieu, and driving local and systemic anti-tumor immunity. ${ }^{*}$ Summarized in Addeo et al. (21) ${ }^{\star}$ Targeting histamine and related cytokines. Discussed in Li et al. (34).

Our secretomic data supports the role of IL-6/sIL-6R $\alpha$ receptor trans-signaling as the key driver of tumor EMT and associated therapy resistance and increased metastatic potential. IL- 6 and its secreted receptor sIL-6R $\alpha$ are increasingly recognized as master cytokines $(40,41)$, upstream of a wide array of inflammatory processes, including pathologies as diverse as cytokine release syndrome (42), acute allograft rejection (43), rheumatoid arthritis (44), asbestosis (45) and cachexia (46). Complexes of soluble 
IL-6/IL-6R $\alpha$ elicit responses from gp130-expressing cells that lack the complete IL- 6 receptor by a process known as trans-signaling $(40,47)$. Figure 1A and our findings in NSCLC-associated MPE (7) reveal a profound degree of cytokine-chemokine polarization dominated by $\mathrm{ng} / \mathrm{mL}$ concentrations of IL-6/sIL6R $\alpha$. Neutralizing pleural IL-6 or IL-6R $\alpha$ activity with therapeutic antibodies may not only diminish IL-6-driven aggressive tumor behavior associated with EMT (48) (Figure 1B), but may also reverse downstream negative regulation of tumor-specific immune effector responses, thereby enhancing the efficacy of other immune oncology therapies. Although long-term antagonism of IL-6R $\alpha$ is immunosuppressive (49), single dose exposure has been shown to break the cytokine storm associated with CAR-T therapy without compromising effector responses (50) or incurring serious adverse events (51). Intra-cavitary administration of anti-IL- 6 or anti-IL-6R $\alpha$ may likewise be expected to exert profound effects on the pleural or peritoneal environments.

Histamine has been shown to play a role in conferring resistance to immunotherapy. $\mathrm{H} 1$ antihistamine therapy counteracts histamine-mediated immunosuppression by counteracting M2 macrophage polarization and promoting CD8+ effector $\mathrm{T}$ cell responses (34). In the intracavitary space, cytokines such as IL-4 and IL-5 that are elevated in MPE (Figure 1) and are central to the allergy cascade (52) and may provide additional targets of therapy.

The pleura and peritoneum are common sites of metastasis for a wide variety of cancers. Their unique physiology makes them ideal tumor sanctuaries that promote aggressive behavior while inhibiting immune effector responses. The contained nature of these spaces also presents an opportunity to therapeutically manipulate the tumor environment in ways that are not possible for other metastatic lesions. There is a wealth of agents available for combination intra-cavitary therapy (Figure 2B), many of which have already shown some activity as single agents. We argue that combination therapies designed to condition the maladaptive cavitary environment, reverse EMT and stimulate immune priming locally and systemically will succeed where single agents have ultimately failed. However,

\section{REFERENCES}

1. Zamboni MM, da Silva CTJr., Baretta R, Cunha ET, Cardoso GP. Important Prognostic Factors for Survival in Patients With Malignant Pleural Effusion. BMC Pulm Med (2015) 15:29-. doi: 10.1186/s12890-015-0025-z

2. Directors ATSBo. Management of Malignant Pleural Effusions. Am J Respir Crit Care Med (2000) 162(5):1987-2001. doi: 10.1164/ajrccm.162.5.ats8-00

3. Clive AO, Kahan BC, Hooper CE, Bhatnagar R, Morley AJ, Zahan-Evans N, et al. Predicting Survival in Malignant Pleural Effusion: Development and Validation of the LENT Prognostic Score. Thorax (2014) 69(12):1098-104. doi: 10.1136/thoraxjnl-2014-205285

4. Ayantunde AA, Parsons SL. Pattern and Prognostic Factors in Patients With Malignant Ascites: A Retrospective Study. Ann Oncol (2007) 18(5):945-9. doi: 10.1093/annonc/mdl499

5. Hodge C, Badgwell BD. Palliation of Malignant Ascites. J Surg Oncol (2019) 120(1):67-73. doi: 10.1002/jso.25453

6. Franke AJ, Iqbal A, Starr JS, Nair RM, George TJ. Management of Malignant Bowel Obstruction Associated With GI Cancers. J Oncol Pract (2017) 13 (7):426-34. doi: 10.1200/JOP.2017.022210

7. Donnenberg AD, Luketich JD, Donnenberg VS. Secretome of Pleural Effusions Associated With Non-Small Cell Lung Cancer (NSCLC) and the devil is in the details, and the challenge will be to design and implement the most agile adaptive therapeutic trials (53) designed to determine the safest and most effective therapeutic combinations and dosing schedules.

\section{DATA AVAILABILITY STATEMENT}

The raw data supporting the conclusions of this article will be made available by the authors, without undue reservation.

\section{AUTHOR CONTRIBUTIONS}

$\mathrm{AD}$ and VD wrote the manuscript, created and analyzed the data. PW contributed the section on peritoneal malignancies and edited the manuscript. JL provided samples and contributed to the discussion and ideas. DB contributed to the discussion and ideas. All authors contributed to the article and approved the submitted version.

\section{FUNDING}

This work was supported by BC032981, BC044784, W81XWH-121-0415 and BC132245_W81XWH-14-0258 from the Department of Defense, National Cancer Institute (NCI) grant R21 CA191647, MetaVivor FP00002718, the Glimmer of Hope Foundation, the Pennsylvania Breast Cancer Coalition, and the David Downing Fund. The Hillman Cancer Center Luminex Facility is supported by Cancer Center Support Grant P30CA047904.

\section{ACKNOWLEDGMENTS}

The authors would like to thank Bosko Popov and Denise Prosser for their excellent technical assistance.

Malignant Mesothelioma: Therapeutic Implications. Oncotarget (2019) 10 (60):6456-65. doi: 10.18632/oncotarget.27290

8. Donnenberg ADDVS.. The Secretome of Malignant Pleural Effusions: Clues to Targets of Therapy. Leukemia Res (2019) 85:S11-2. doi: 10.1016/S01452126(19)30222-X

9. Koopmans T, Rinkevich Y. Mesothelial to Mesenchyme Transition as a Major Developmental and Pathological Player in Trunk Organs and Their Cavities. Commun Biol (2018) 1(1):170. doi: 10.1038/s42003-018-0180-x

10. Amasheh S, Markov AG, Volgin GN, Voronkova MA, Yablonsky PK, Fromm M. Barrier Function of Human Pleura Mesothelium is Constituted by Tight Junctions. FASEB J (2011) 25(1_supplement):1036.3-.3. doi: 10.1096/fasebj. 25.1_supplement.1036.3

11. Donnenberg AD, Luketich JD, Dhupar R, Donnenberg VS. Treatment of Malignant Pleural Effusions: The Case for Localized Immunotherapy. J ImmunoTher Cancer (2019) 7(1):110. doi: 10.1186/s40425-019-0590-4

12. Goey SH, Eggermont AM, Punt CJ, Slingerland R, Gratama JW, Oosterom R, et al. Intrapleural Administration of Interleukin 2 in Pleural Mesothelioma: A Phase I-II Study. Br J Cancer (1995) 72(5):1283-8. doi: 10.1038/bjc.1995.501

13. Ruf $\mathrm{P}$, Kluge $M$, Jager $M$, Burges A, Volovat C, Heiss MM, et al. Pharmacokinetics, Immunogenicity and Bioactivity of the Therapeutic Antibody Catumaxomab Intraperitoneally Administered to Cancer Patients. 
Br J Clin Pharmacol (2010) 69(6):617-25. doi: 10.1111/j.1365-2125.2010. 03635. $\mathrm{x}$

14. Sebastian M, Kiewe P, Schuette W, Brust D, Peschel C, Schneller F, et al. Treatment of Malignant Pleural Effusion With the Trifunctional Antibody Catumaxomab (Removab) (Anti-EpCAM $\times$ Anti-CD3): Results of a Phase 1/2 Study. J Immunother (2009) 32(2):195-202. doi: 10.1097/CJI. 0b013e318195b5bb

15. Sahai E, Astsaturov I, Cukierman E, DeNardo DG, Egeblad M, Evans RM, et al. A Framework for Advancing Our Understanding of Cancer-Associated Fibroblasts. Nat Rev Cancer (2020) 20(3):174-86. doi: 10.1038/s41568-0190238-1

16. Telvi L, Jaubert F, Eyquem A, Andreux JP, Labrousse F, Chretien J. Study of Immunoglobulins in Pleura and Pleural Effusions. Thorax (1979) 34(3):38992. doi: 10.1136/thx.34.3.389

17. Hewitt SL, Bailey D, Zielinski J, Apte A, Musenge F, Karp R, et al. Intratumoral IL12 mRNA Therapy Promotes TH1 Transformation of the Tumor Microenvironment. Clin Cancer Res (2020) 26(23):6284-98. doi: 10.1158/1078-0432.CCR-20-0472

18. Di Trani CA, Fernandez-Sendin M, Cirella A, Segués A, Olivera I, Bolaños E, et al. Advances in mRNA-Based Drug Discovery in Cancer Immunotherapy. Expert Opin Drug Discov (2021) 17(1):1-13. doi: 10.1080/17460441. 2021.1978972

19. Pennathur A, Abbas G, Christie N, Landreneau R, Luketich JD. Video Assisted Thoracoscopic Surgery and Lobectomy, Sublobar Resection, Radiofrequency Ablation, and Stereotactic Radiosurgery: Advances and Controversies in the Management of Early Stage non-Small Cell Lung Cancer. Curr Opin Pulmonary Med (2007) 13(4):267-70. doi: 10.1097/ MCP.0b013e3281c61a85

20. Chang E, Alexander HR, Libutti SK, Hurst R, Zhai S, Figg WD, et al. Laparoscopic Continuous Hyperthermic Peritoneal Perfusion1. J Am Coll Surgeons (2001) 193(2):225-9. doi: 10.1016/S1072-7515(01)00980-2

21. Muller PY, Milton M, Lloyd P, Sims J, Brennan FR. The Minimum Anticipated Biological Effect Level (MABEL) for Selection of First Human Dose in Clinical Trials With Monoclonal Antibodies. Curr Opin Biotechnol (2009) 20(6):722-9. doi: 10.1016/j.copbio.2009.10.013

22. Hu CY, Zhang YH, Wang T, Chen L, Gong ZH, Wan YS, et al. Interleukin-2 Reverses CD8(+) T Cell Exhaustion in Clinical Malignant Pleural Effusion of Lung Cancer. Clin Exp Immunol (2016) 186(1):106-14. doi: 10.1111/cei.12845

23. Sterman DH, Recio A, Carroll RG, Gillespie CT, Haas A, Vachani A, et al. A Phase I Clinical Trial of Single-Dose Intrapleural IFN- $\beta$ Gene Transfer for Malignant Pleural Mesothelioma and Metastatic Pleural Effusions: High Rate of Antitumor Immune Responses. Clin Cancer Res (2007) 13(15):4456-66. doi: 10.1158/1078-0432.CCR-07-0403

24. Zeltsman M, Mayor M, Jones DR, Adusumilli PS. Surgical Immune Interventions for Solid Malignancies. Am J Surg (2016) 212(4):682-90.e5. doi: 10.1016/j.amjsurg.2016.06.008

25. Chandran SS, Somerville RPT, Yang JC, Sherry RM, Klebanoff CA, Goff SL, et al. Treatment of Metastatic Uveal Melanoma With Adoptive Transfer of Tumour-Infiltrating Lymphocytes: A Single-Centre, Two-Stage, Single-Arm, Phase 2 Study. Lancet Oncol (2017) 18(6):792-802. doi: 10.1016/S1470-2045 (17)30251-6

26. Murthy V, Katzman D, Sterman DH. Intrapleural Immunotherapy: An Update on Emerging Treatment Strategies for Pleural Malignancy. Clin Respir J (2019) 13(5):272-9. doi: 10.1111/cri.13010

27. Addeo A, Friedlaender A, Giovannetti E, Russo A, de Miguel-Perez D, Arrieta $\mathrm{O}$, et al. A New Generation of Vaccines in the Age of Immunotherapy. Curr Oncol Rep (2021) 23(12):137. doi: 10.1007/s11912-021-01130-x

28. Miao L, Zhang Y, Huang L. mRNA Vaccine for Cancer Immunotherapy. Mol Cancer (2021) 20(1):41. doi: 10.1186/s12943-021-01335-5

29. Eisenhauer EA, Therasse P, Bogaerts J, Schwartz LH, Sargent D, Ford R, et al. New Response Evaluation Criteria in Solid Tumours: Revised RECIST Guideline (Version 1.1). Eur J Cancer (2009) 45(2):228-47. doi: 10.1016/ j.ejca.2008.10.026

30. Edwards RP, Gooding W, Lembersky BC, Colonello K, Hammond R, Paradise C, et al. Comparison of Toxicity and Survival Following Intraperitoneal Recombinant Interleukin-2 for Persistent Ovarian Cancer After Platinum: Twenty-Four-Hour Versus 7-Day Infusion. J Clin Oncol (1997) 15(11):3399407. doi: 10.1200/JCO.1997.15.11.3399
31. Sehouli J, Pietzner K, Wimberger P, Vergote I, Rosenberg P, Schneeweiss A, et al. Catumaxomab With and Without Prednisolone Premedication for the Treatment of Malignant Ascites Due to Epithelial Cancer: Results of the Randomised Phase IIIb CASIMAS Study. Med Oncol (2014) 31(8):76. doi: 10.1007/s12032-014-0076-7

32. Elkadi D, Wiernik PH, Tong TR. Resolution of Massive Pleural Effusion Due to Lymphoma With Intrapleural Interleukin-2. Am J Hematol (2010) 85 (9):711-2. doi: 10.1002/ajh.21604

33. Santini D, Zeppola T, Russano M, Citarella F, Anesi C, Buti S, et al. PD-1/PDL1 Checkpoint Inhibitors During Late Stages of Life: An Ad-Hoc Analysis From a Large Multicenter Cohort. J Transl Med (2021) 19(1):270. doi: 10.1186/s12967-021-02937-9

34. Li H, Xiao Y, Li Q, Yao J, Yuan X, Zhang Y, et al. The Allergy Mediator Histamine Confers Resistance to Immunotherapy in Cancer Patients via Activation of the Macrophage Histamine Receptor H1. Cancer Cell (2022) 40(1):36-52.e9. doi: 10.1016/j.ccell.2021.11.002

35. Ekeke CN, Russell KL, Joubert K, Bartlett DL, Luketich JD, Soloff AC, et al. Fighting Fire With Fire: Oncolytic Virotherapy for Thoracic Malignancies. Ann Surg Oncol (2021) 28(5):2715-27. doi: 10.1245/s10434-020-09477-4

36. Urban-Wojciuk Z, Khan MM, Oyler BL, Fåhraeus R, Marek-Trzonkowska N, Nita-Lazar A, et al. The Role of TLRs in Anti-Cancer Immunity and Tumor Rejection. Front Immunol (2019) 10(2388). doi: 10.3389/fimmu.2019.02388

37. Bone G. Postoperative Empyema and Survival in Lung Cancer. $\mathrm{Br} \mathrm{Med} \mathrm{J}$ (1973) 1(5852):504-5. doi: 10.1136/bmj.1.5852.504-a

38. Ruckdeschel JC, Codish SD, Stranahan A, McKneally MF. Postoperative Empyema Improves Survival in Lung Cancer. Documentation and Analysis of a Natural Experiment. N Engl J Med (1972) 287(20):1013-7. doi: 10.1056/ NEJM197211162872004

39. Brombacher F, Kastelein RA, Alber G. Novel IL-12 Family Members Shed Light on the Orchestration of Th1 Responses. Trends Immunol (2003) 24 (4):207-12. doi: 10.1016/S1471-4906(03)00067-X

40. Uciechowski P, Dempke WCM. Interleukin-6: A Masterplayer in the Cytokine Network. Oncology (2020) 98(3):131-7. doi: 10.1159/000505099

41. Scheller J, Chalaris A, Schmidt-Arras D, Rose-John S. The Pro- and AntiInflammatory Properties of the Cytokine Interleukin-6. Biochim Biophys Acta (BBA) Mol Cell Res (2011) 1813(5):878-88. doi: 10.1016/j.bbamcr.2011.01.034

42. Maude SL, Barrett D, Teachey DT, Grupp SA. Managing Cytokine Release Syndrome Associated With Novel T Cell-Engaging Therapies. Cancer J (2014) 20(2):119-22. doi: 10.1097/PPO.0000000000000035

43. Iacono A, Dauber J, Keenan R, Spichty K, Cai J, Grgurich W, et al. Interleukin 6 and Interferon-Gamma Gene Expression in Lung Transplant Recipients With Refractory Acute Cellular Rejection: Implications for Monitoring and Inhibition by Treatment With Aerosolized Cyclosporine. Transplantation (1997) 64(2):263-9. doi: 10.1097/00007890-199707270-00015

44. Davies R, Choy E. Clinical Experience of IL-6 Blockade in Rheumatic Diseases -Implications on IL-6 Biology and Disease Pathogenesis. Semin Immunol (2014) 26(1):97-104. doi: 10.1016/j.smim.2013.12.002

45. Doré P, Lelièvre E, Morel F, Brizard A, Fourcin M, Clemént C, et al. IL-6 and Soluble IL-6 Receptors (sIL-6R and Sgp130) in Human Pleural Effusions: Massive IL-6 Production Independently of Underlying Diseases. Clin Exp Immunol (1997) 107(1):182-8. doi: 10.1046/j.1365-2249.1997.d01-889.x

46. Rupert JE, Narasimhan A, Jengelley DHA, Jiang Y, Liu J, Au E, et al. TumorDerived IL-6 and Trans-Signaling Among Tumor, Fat, and Muscle Mediate Pancreatic Cancer Cachexia. J Exp Med (2021) 218(6). doi: 10.1084/ jem. 20190450

47. Lo C-W, Chen M-W, Hsiao M, Wang S, Chen C-A, Hsiao S-M, et al. IL-6 Trans-Signaling in Formation and Progression of Malignant Ascites in Ovarian Cancer. Cancer Res (2011) 71(2):424. doi: 10.1158/0008-5472.CAN-10-1496

48. Lu H, Clauser KR, Tam WL, Frose J, Ye X, Eaton EN, et al. A Breast Cancer Stem Cell Niche Supported by Juxtacrine Signalling From Monocytes and Macrophages. Nat Cell Biol (2014) 16(11):1105-17. doi: 10.1038/ncb3041

49. Pawar A, Desai RJ, Solomon DH, Santiago Ortiz AJ, Gale S, Bao M, et al. Risk of Serious Infections in Tocilizumab Versus Other Biologic Drugs in Patients With Rheumatoid Arthritis: A Multidatabase Cohort Study. Ann Rheumatic Dis (2019) 78(4):456. doi: 10.1136/annrheumdis-2018-214367

50. Davila ML, Riviere I, Wang X, Bartido S, Park J, Curran K, et al. Efficacy and Toxicity Management of 19-28z CAR T Cell Therapy in B Cell Acute Lymphoblastic Leukemia. Sci Trans Med (2014) 6(224):224ra25. 
51. Xu X, Han M, Li T, Sun W, Wang D, Fu B, et al. Effective Treatment of Severe COVID-19 Patients With Tocilizumab. ChinaXiv (2020) 00026v1. doi: 10.1073/pnas.2005615117

52. Krouwels FH, Hol BE, Lutter R, Bruinier B, Bast A, Jansen HM, et al. Histamine Affects Interleukin-4, Interleukin-5, and Interferon-Gamma Production by Human T Cell Clones From the Airways and Blood. Am J Respir Cell Mol Biol (1998) 18(5):721-30. doi: 10.1165/ajrcmb.18.5.2909

53. Park JJ, Thorlund K, Mills EJ. Critical Concepts in Adaptive Clinical Trials. Clin Epidemiol (2018) 10:343-51. doi: 10.2147/CLEP.S156708

Conflict of Interest: The authors declare that the research was conducted in the absence of any commercial or financial relationships that could be construed as a potential conflict of interest.
Publisher's Note: All claims expressed in this article are solely those of the authors and do not necessarily represent those of their affiliated organizations, or those of the publisher, the editors and the reviewers. Any product that may be evaluated in this article, or claim that may be made by its manufacturer, is not guaranteed or endorsed by the publisher.

Copyright (c) 2022 Donnenberg, Wagner, Luketich, Bartlett and Donnenberg. This is an open-access article distributed under the terms of the Creative Commons Attribution License (CC BY). The use, distribution or reproduction in other forums is permitted, provided the original author(s) and the copyright owner(s) are credited and that the original publication in this journal is cited, in accordance with accepted academic practice. No use, distribution or reproduction is permitted which does not comply with these terms. 